\title{
Multiphase Flow Analysis in an Oil-injected Twin Screw Compressor
}

\author{
Nausheen Basha, Sham Rane, Ahmed Kovacevic \\ City, University of London \\ Northampton Square, London, UK \\ Nausheen.basha@city.ac.uk,sham.rane@city.ac.uk, a.kovacevic@city.ac.uk
}

\begin{abstract}
Oil injected twin screw compressors are widely used in industry for gas compression because of their high volumetric efficiency and reliability. The injected oil has a significant influence on the performance of these compressors. Only an optimum quantity of oil supply would give best performance at a specified operating condition. Therefore, it is essential to determine the distribution of oil in the working chamber and thereby the compressor's performance. Using Computational Fluid Dynamics (CFD) to solve two-phase flow in deforming domains is numerically challenging due to the complex interactions between air and oil in rotating machines. In this paper, two Eulerian-Eulerian multiphase models have been investigated. Namely, inhomogeneous and homogeneous model with free surface modelling have been investigated for their stability, oil-air distribution and accuracy of compressor performance results. Deforming rotor mesh needed for the simulation is generated by SCORG ${ }^{\mathrm{TM}}$ grid generator while two-phase transient flow is solved with ANSYS CFX solver. Results obtained from the simulation have been compared with the measured data of mass flow rate of gas, indicated power and temperatures. Inhomogeneous model predicts data closer to the experimental results and an expected distribution of oil in the rotor tip clearances. For a high mesh deformation of a working domain in twin screw compressor, the inhomogeneous approach also provides better stability of the solver, but this is at an added cost of calculation time. In future, better initialisation and solver parameters with oil droplet characterisation will be required to further improve the model.
\end{abstract}

Keywords: Twin Screw Compressor, Multiphase, CFD, Oil-injection, ANSYS CFX, Eulerian, SCORG ${ }^{\mathrm{TM}}$.

\section{Introduction}

Oil-injected screw compressors are used in industry whenever traces of oil are allowed in the compressed gas as they achieve higher pressures and much lower discharge temperatures than oil free compressors. Oil serves the purpose of cooling the gas inside the compressor chamber, sealing the clearance gaps and lubrication the rotors and bearings. Also, presence of oil reduces noise in screw compressors.

Since screw compressor is a machine with tight clearances, oil is essential for its effective running and prevention of seizure. Approximately $90 \%$ of injected oil is used for cooling, 9\% for sealing and $1 \%$ for lubrication [1]. Though oil improves the performance of the compressor significantly, it also adds some power losses. The power losses due to oil are caused by shear of oil in clearances and surge of oil when the oil film is thicker than the clearance height. This results in hydraulic frictional losses and momentum losses due to acceleration of oil on inner surfaces and during the oil injection process. Therefore, oil injection should be optimised to achieve a better performing screw compressor. In order to study the losses associated with oil in the compression chamber, it is necessary to understand the distribution of oil. It is extremely difficult, if not impossible to experimentally determine oil distribution in oil injected screw machines. Previously, thermodynamic chamber models were conveniently used to predict compressor performance [2], but these are not capable of determining distribution of oil inside the machine. On the other side, Computational Fluid Dynamics (CFD) provides an excellent insight towards the flow pattern of oil and gas inside positive displacement screw machine.

Kovacevic [3] [4] [5] made a breakthrough in CFD modelling of screw machines by developing a methodology to produce block structured grids for deforming domains using algebraic grid generation. Based on this, a standalone programme SCORG (Screw Compressor Rotor Grid Generator) is developed to generate and supply a numerical mesh and relevant parameters to commercial CFD solvers. Then, SCORG was tested with the commercial solver COMET of CD Adapco using Euler-Lagrange multiphase flow model for oil injected air compressor and for the very first time it was possible to visualise flow characteristics within screw compressor chamber. The effects of mass and momentum change of the dispersed phase, normally oil or water, on the continuous phase of gas in this model were accounted for through source terms in conservation equations. These source terms are programmed as user subroutines through very open platform of 
software Comet [5]. Not only the computational modelling methodology was established, also the modelling results were closer to experimental results which encouraged further research in this direction. Various developments on improving the quality of grid in deforming domains and enabling multiphase flow calculation with other commercial solvers took place in years that followed. For instance, Vande Voorde and Vierendeels [6], using the same domain decomposition strategy, developed numerical procedure to produce grids through solution of a Laplace potential equation and achieve better cell orthogonality. These developed grids were applied to study the mass flow rate through leakages in an oil injected expander in ANSYS FLUENT along with User Defined Functions (UDF) for handling the grid movement with varying time step and Eulerian multiphase model [7]. However, the results from the model were not validated with the experimental data. Rane and Kovacevic applied numerical orthogonalisation and smoothing to grids generated by analytical transfinite interpolation and eliminated the non-conformal interface between the rotor domains resulting in a single domain structured grid for the rotors [8]. The procedure significantly improved orthogonality of the mesh but retained the speed of calculation of analytical grid generation. The conformal interface adds to accuracy. The predicted indicated power and flow rates of an oil-free compressor are within $1 \%$ and $2 \%$ respectively in comparison to experimentally obtained values [9]. Firstly, the improved grid quality of a single domain grid allows use of SCORG meshes with any arbitrary CFD solver and secondly, allows accurate analysis of complex physical phenomenon such as multiphase flows since the nonphysical errors in handling sliding and stretching boundary between the rotor domains are avoided.

In the recent study, Volume of Fluids (VOF) model was used to simulate flow in oil injected compressor with another commercial solver Pumplinx in which the oil was assumed to be uniformly mixed with the gas at the compressor inlet with up to 57\% mass fraction [10]. The CFD results established that the compressor under the same operating condition has a $23 \%$ increase in gas mass flow rate, $74 \%$ reduction in gas temperature rise at the outlet and $12 \%$ reduction in rotor power with oil injection compared with the oil free application. Rane et al [1] modelled oil injection in ANSYS CFX with inhomogeneous multiphase model and validated the predicted results with data from screw compressor tested at 6000 and 8000 RPM. In addition to this, close interaction of oil with gate rotors is visualised. It could be observed that the oil injection starts with oil as spray, which then leads to accumulation of a film on the rotor surfaces, this film grows and spreads until the injection nozzle is cut off from the compressor chamber by rotor trailing lobes.

The study by Rane [1] showed that the inhomogeneous model in ANSYS CFX gives expected results, but this multiphase model in its nature is computationally intensive and expensive. Therefore, in this study a simpler version of Eulerian-Eulerian modelling which is homogeneous model and with the need to track the oil interface free surface modelling can be investigated for its comparative accuracy and feasibility. If the homogeneous model proves to be accurate enough then this can reduce computational time significantly which will result in a quicker compressor design development time. The mathematical differences between inhomogeneous and homogeneous are explained in the next section.

\section{Governing Equations for Modelling of Multiphase Flows}

Modelling multiphase flows introduces complexity in CFD analysis as it is often limited by the computational capacity of the available computers and programs. The most widely used methods for multiphase modelling in commercial CFD solvers are Euler-Euler and Euler-Lagrangian approach. In the former the flow phases are treated as interpenetrating continuous media while in the later approach gas is assumed as a continuous medium while liquid is treated as dispersed phase. This means that in the Euler-Lagrange methods some special treatment needs to be applied to account for the influence of the mass and momentum of liquid on the behaviour of a continuous phase.

Therefore, more often Euler-Euler approach is used in treatment of multiphase flows in screw machines. Two different models are available under Euler-Euler multiphase flow simulation in ANSYS CFX, namely homogeneous and inhomogeneous model. Homogeneous modelling is a computationally cheaper since the volume of each phase is used to determine the fluid mixture properties which are then solved using one set of equations for the mixture. If including free surface model, it would be comparable to Volume of Fluids (VOF) model developed by Hirt and Nichols [11]. On the other side, inhomogeneous model is computationally intensive since the transport equations are solved for every single phase considering its volume fraction in the medium and the separate solutions of the phase are linked at the interface through mass and momentum transfer equations.

Mass conservation equation of an inhomogeneous viscous flow of two phases $\alpha$ and $\beta$, can be describes as [12]: 


$$
\frac{\partial}{\partial t}(r \rho)+\frac{\partial}{\partial x_{i}}\left(r \rho u_{i}\right)=m+\Gamma^{\alpha \beta}
$$

where $\Gamma^{\alpha \beta}$ is mass transfer from $\alpha$ to $\beta$ phase (vice-versa for the term $\Gamma^{\beta \alpha}$ ), $m$ is mass sources, $\rho$ is density, $r$ is volume fraction and $\mathrm{u}_{\mathrm{i}}$ is velocity of phase $\alpha$. The corresponding equation for conservation of momentum is

$$
\frac{\partial}{\partial t}\left(r \rho u_{i}\right)+\frac{\partial}{\partial x_{j}}\left(r \rho u_{i} u_{j}\right)=-r \frac{\partial p}{\partial x_{i}}+\frac{\partial\left(r \tau_{i j}\right)}{\partial x_{j}}+M^{\Gamma}+M^{\alpha}+b_{i}
$$

where $b_{i}$ are body forces, $\mathbf{M}^{\alpha}$ considers the forces on the interface caused by the presence of phase $\beta$ this includes forces such as drag force, lift force due to fluid rotation and wall lubrication, $\mathrm{u}_{\mathrm{j}}$ is velocity of phase $\beta$, $\mathrm{p}$ is pressure shared by the phases, $\mu$ is the dynamic viscosity, the term $\mathrm{M}^{\Gamma}$ is the momentum transfer caused by the mass transfer and the stress tensor $\tau_{\mathrm{ij} \text {. }}$

$$
M^{\Gamma}=\Gamma^{\alpha \beta} u_{i}^{\beta}-\Gamma^{\beta \alpha} u_{i}^{\alpha}
$$

Equations 1 and 2 are computationally intensive since they are solved for each phase individually. Say for a two phase flow with inhomogeneous approach, three momentum equations need to be solved for the first phase in $x, y$ and ,z directions and additional three momentum equations for the second phase. A simplification to (1) and (2) is given with the homogeneous multiphase flow model. Here, the relative slip between the phases is neglected [13], resulting in no consideration for $\mathrm{M}^{\alpha}$ in equation (2). Due to this, the interface momentum transfer $\left(\mathrm{M}^{\Gamma}\right)$ is large, but the velocity for both phases is equal. Hence, considering equation (3), the interface momentum transfer can be neglected from equation (2). The set of equations with this simplification, as shown in [12]is:

$$
\begin{gathered}
\frac{\partial(r \rho)}{\partial t}+\frac{\partial}{\partial x_{i}}\left(r \rho u_{i}\right)=0 \\
\frac{\partial}{\partial t}\left(\rho U_{i}\right)+\frac{\partial}{\partial x_{j}}\left(r \rho u_{i} u_{j}\right)=-\frac{\partial p}{\partial x_{i}}+\mu \frac{\partial \tau_{i j}}{\partial x_{j}}+b_{i} \\
\mu=\sum_{l=1}^{2} r_{l} \mu_{l} \\
\rho=\sum_{l=1}^{2} r_{l} \rho_{l}
\end{gathered}
$$

1 in above equation represents number of phases. Therefore, in the homogeneous model, transport quantities are shared and solved within the single set of transport equations. Solving a single set of momentum equation reduces solver time significantly. The effect of this approach with the common flow field shared by all fluids will be explored in this paper for a case of oil injected compressor. In order to capture the heat transfer between the phases with inhomogeneous as well as homogeneous model, equations of energy selected for both cases are same [14].

$$
\frac{\partial}{\partial t}\left(r \rho h_{\alpha}\right)+\nabla \cdot\left(r\left(\rho U_{\alpha} h_{\alpha}-\delta_{\alpha} \nabla T_{\alpha}\right)\right)=\sum_{l=1}^{2}\left(\Gamma^{\alpha \beta} h_{\beta}-\Gamma^{\beta \alpha} h_{\alpha}\right)+Q_{\alpha \beta}+S_{\alpha}
$$

Here, $\mathrm{h}_{\alpha}, \mathrm{T}_{\alpha}$ and $\delta_{\alpha}$ denote static enthalpy, temperature and thermal conductivity of the phase $\alpha . \mathrm{T}_{\beta}$ represents temperature for phase $\beta$ and $S_{\alpha}$ describes external heat sources (momentum, continuity etc.). $Q_{\alpha \beta}$ is the heat transfer 
through the interface between the phases which takes into account heat transfer coefficient, interface area and the temperature difference.

\section{Case Study}

\subsection{Compressor Specification}

An oil-injected twin screw compressor with axial and radial suction port and radial discharge port is analysed in this paper. The lobe combination is $4-5$ with ' $\mathrm{N}$ rotor profile'. The rotor centre distance is $98 \mathrm{~mm}$, the outer diameter of the male rotor is $130.445 \mathrm{~mm}$ and the outer diameter of the female rotor is $105.245 \mathrm{~mm}$. The length of the rotors is $202.55 \mathrm{~mm}$ with the male rotor wrap angle of $306.47^{\circ}$ and volume index of 3.6. The nominal interlobe, radial and axial clearances are $50 \mu \mathrm{m}$. The compressor operates at 6000RPM and the discharge pressure of 8.0 bar. The suction is at atmospheric conditions. The compressor performance was measured at City, University of London's test rig as show in Fig. 1a. The compressor is driven by a $75 \mathrm{~kW}$ motor with the speed adjusted by the variable frequency drive. The discharge pressure is controlled using a check valve and the gas flow rate is measured using an orifice meter. Oil is injected using the discharge pressure and its temperature is controlled by water cooled heat exchanger. This experimentally obtained data will be used for comparison with the values predicted by CFD.

\subsection{CFD Case Setup}

The CAD model of the flow domains in the compressor used for CFD analysis is shown in Fig. 1b. The numerical grid used for CFD calculations is shown in Fig. 1c. It consists of the deforming single domain mesh for the rotors which is formed of hexahedral cells and generated by SCORG ${ }^{\mathrm{TM}}$. It is confirmed in literature that a numerical grid which avoids mismatch caused by a non-conformal boundary leads to better accuracy, stability and no loss of flux between the interfaces [9].

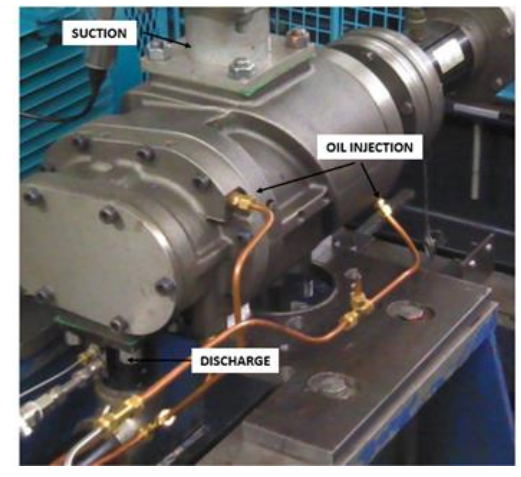

a

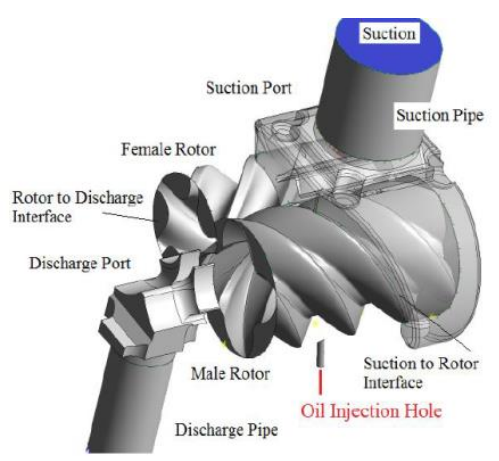

b

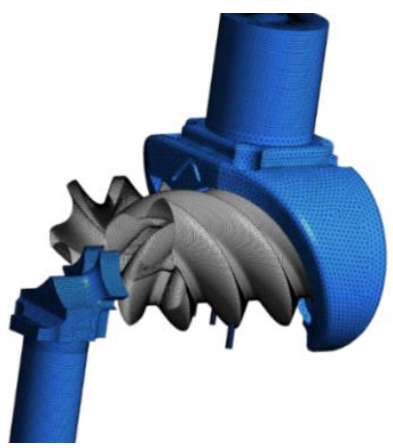

$\mathrm{c}$

Fig. 1: (a) Compressor in experimental test rig (b) CAD model and (c) Numerical grid of the flow domain.

This compressor has four oil injection ports. The injection ports as well as the suction and discharge ports are connected to the rotor domain by fluid-fluid interface. This type of interface is used in order to enable conservative flux for all transport quantities through the interface. All numerical meshes required to fully describe the rotor rotation are created in advance of CFD simulation. Although important in reality, the axial end clearance gap is not included in the CFD model for this study to allow reduction of the numerical grid size. Details of the grid size and type are presented in Table 1. ANSYS CFX solver is based on vertex centred computational cell structure and pressure based coupled solution algorithm. Properties of air and oil considered in ANSYS CFX are tabulated in Table 2. Also, the main settings in the solver are listed in Table 3. 
Table 1: Mesh Statistics.

\begin{tabular}{|c|c|c|c|}
\hline Domain & Cell structure & Node Count & Cell Count \\
\hline Rotor & Hexahedral & 468677 & 406368 \\
\hline Suction Port & Tetra+Hex & 119058 & 203255 \\
\hline Discharge Port & Tetra+Hex & 98521 & 253095 \\
\hline Oil Injection Port & Hexahedral & 28340 & 28340 \\
\hline
\end{tabular}

Table 2: Air and oil properties.

\begin{tabular}{|c|c|c|c|c|}
\hline Phase & $\begin{array}{c}\text { Density } \\
\left(\mathbf{K g} / \mathbf{m}^{\mathbf{3}}\right)\end{array}$ & $\begin{array}{c}\text { Specific Heat } \\
\text { Capacity }(\mathbf{J} / \mathbf{k g ~ K})\end{array}$ & $\begin{array}{c}\text { Dynamic Viscosity } \\
(\mathbf{k g} / \mathbf{m s})\end{array}$ & $\begin{array}{c}\text { Thermal Conductivity } \\
(\mathbf{W} / \mathbf{m ~ K})\end{array}$ \\
\hline Air & 1.225 & 1.0044 & $1.831 \times 10^{-5}$ & $2.61 \times 10^{-2}$ \\
\hline Oil & 800 & 1800.0 & 0.0088 & 0.18 \\
\hline
\end{tabular}

Table 3: CFD solver parameters.

\begin{tabular}{|c|c|}
\hline Criteria & Selection \\
\hline Turbulence model & SST k- $\omega$ \\
\hline Advection Scheme & Upwind \\
\hline Turbulence Scheme & First Order Upwind \\
\hline Transient Scheme & Second Order Backward Euler \\
\hline Inner Coefficients & Up to 5 iterations per time step \\
\hline Convergence Criteria & $5 \mathrm{e}^{-04}$ RMS Residual Level \\
\hline Relaxation Parameters & Solver Relaxation Parameters of 0.05 to achieve stability \\
\hline
\end{tabular}

In addition to the above parameters mentioned in Table 3, an interface length scale of 5 microns is specified for inhomogeneous model. This corresponds to the factor responsible for the momentum transfer between the phases (equation 2 ) in the inhomogeneous model but it is neglected in the homogeneous model. For stability reasons and better initialisation of the case, both discharge pressure and oil mass flow are ramped up to their full values. The discharge pressure is ramped up across 800 time steps and oil mass flow across 400 time steps.

Both inhomogeneous and homogeneous cases are solved with $32 \mathrm{~GB}$ RAM and $3 \mathrm{GHz}$ processer. Comparison is drawn on the solver time and mass imbalance, and is noted down in the Table 4. Overall, solution through homogeneous model is quicker, but it lacks stability. Inhomogeneous case was solved with inlet and outlet of boundary type 'opening', but outlet boundary type was changed to 'out' with homogeneous model to avoid flow pulsations. This improved the simulation stability.

Table 4: Summary on case comparison.

\begin{tabular}{|c|c|c|}
\hline Parameter & Inhomogeneous & Homogeneous \\
\hline Parallel Nodes & 6 & 4 \\
\hline Time step size & $4.16 \times 10^{-6}$ & $4.16 \times 10^{-6}$ \\
\hline Physical time required to solve for 1 time step & 7 mins & 4.4 mins \\
\hline Physical time required for one complete rotation & 18.6 hours & 11.73 hours \\
\hline Number time steps solved & 1600 (10 male rotor rotations) & 1600 (10 male rotor rotations) \\
\hline Physical time to solve complete case & 186.6 hours & 117.3 hours \\
\hline Error in mass imbalance for air phase & Below $9 \%$ & Below 15\% \\
\hline Error in mass imbalance for oil phase & Below 9\% & Below $2 \%$ \\
\hline
\end{tabular}


All results in further sections from both the homogeneous and inhomogeneous approach are compared at same time steps. The air mass imbalance error is higher with homogeneous model while mass imbalance for oil is higher for inhomogeneous model. This is likely due to the fact that in homogeneous model a single mas conservation equation for oil is solved whereas for inhomogeneous model mas conservation for oil and gas are solved separately[15] . Saying that, to determine exact reason for error in mass imbalance might need further investigation.

\section{Results and Discussion}

\subsection{Performance Comparison}

Fig. 2 shows the comparison of the flow rates and indicated powers obtained by integrating CFD results solved with inhomogeneous and homogeneous multiphase models and experimental data. The homogeneous model does not predict the flow rate well compared to the inhomogeneous model. Homogeneous model predicts lower flow rate by $30.6 \%$ and indicated power by $25.8 \%$ whereas inhomogeneous model predicts lower flow rate by $0.5 \%$ and higher power by $3.8 \%$ compared to measured values. The lower flow rate indicates higher leakage across clearance gaps. This may be attributed to the fact that in homogeneous model, air and oil share the same velocity field. Thereby, with higher momentum of the mixture, oil passes through the clearance gaps rather than being effective in sealing action. This scenario combined with the higher temperatures in the compression chamber (Fig. 6b) leads to lower flow rate and indicated power predictions. It has to be noted that, in order to compare with CFD results, the measured shaft power was multiplied with an approximate mechanical efficiency constant of $70 \%$.
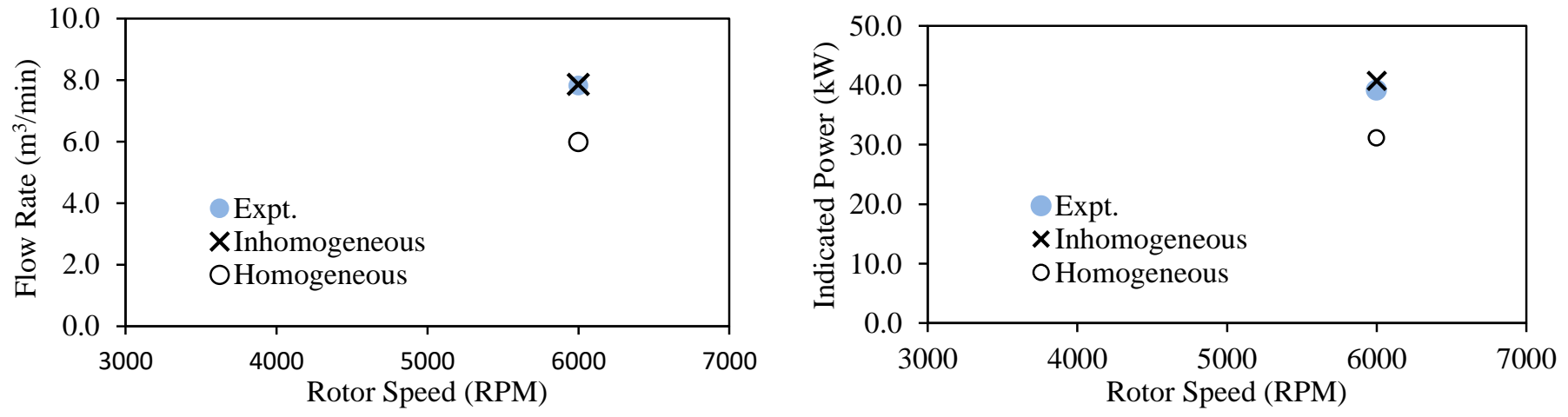

Fig. 2: Flow rate and indicated power against rotor speed.

Fig. 3 shows variation in the interlobe pressure with the main rotor rotation angle. The pressure obtained by both homogeneous and inhomogeneous models are very similar. On average, homogeneous case under predicts pressure by 4.6\% compared to inhomogeneous case. However there are regions with the pressure of homogenous case higher than inhomogeneous especially close to the injection points indicated by the unrealistic pressure distribution. The double pressure peak in the discharge process is observed in the inhomogeneous case which can be associated to the type of boundary conditions set for the inhomogeneous case as opening. This allows reverse flow which is not the case for outlet which is set for the homogeneous case. 


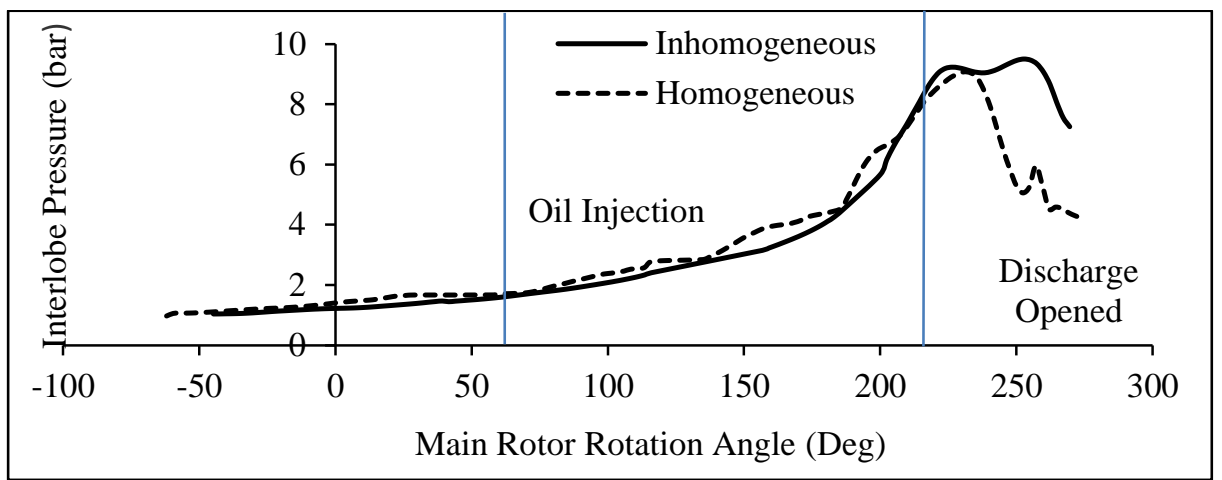

Fig. 3: Pressure variation with male rotor rotation angle.

\subsection{Oil and Temperature Distribution}

The pulsating nature of oil injection is shown in Fig. 4 (Right). The pressure in the oil injection holes is set at around 6.5 barg taking into account 0.5 bar pressure loss in the discharge system. In the inhomogeneous case, the discharge pressure is higher than in the homogeneous case which results in oil flow rate higher by 5.74\%. Fig. 4 (Left) shows the discharge air temperature. In the homogeneous case, the discharge temperature is $17.9{ }^{\circ} \mathrm{C}$ lower than experimentally measured while in the inhomogeneous case it is $8{ }^{\circ} \mathrm{C}$ lower than experiment. This can be attributed to the distribution of oil near the port area. Higher oil volume fraction is observed in the homogeneous model in the cross section through the discharge chamber in Fig. 5b. In the homogeneous model, oil and gas share the same velocity and oil quickly trails out the compressor resulting in a short residence time and higher compression chamber temperature.
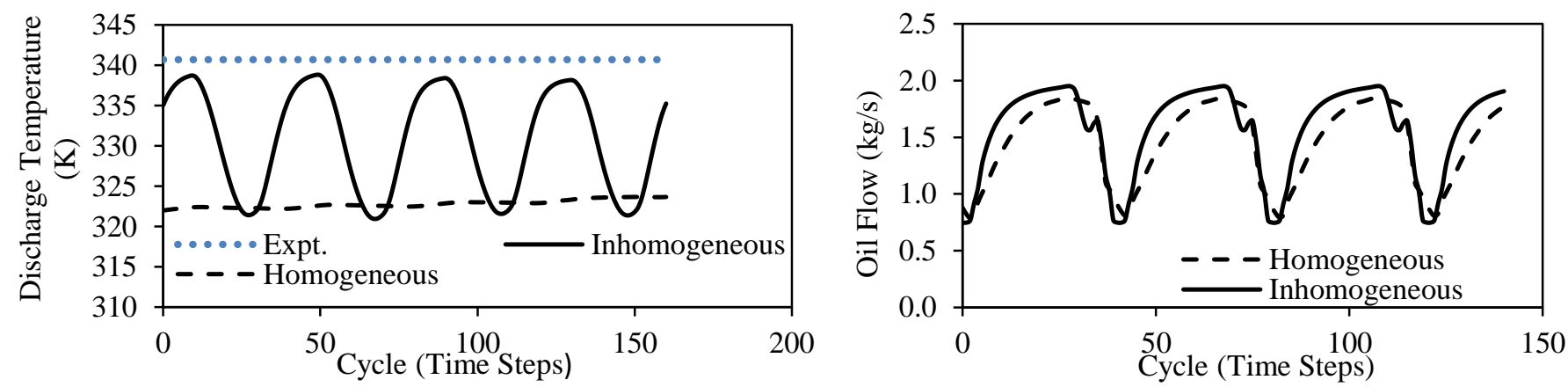

Fig. 4: Discharge temperature in a rotation cycle and Flow of oil in a rotation cycle.

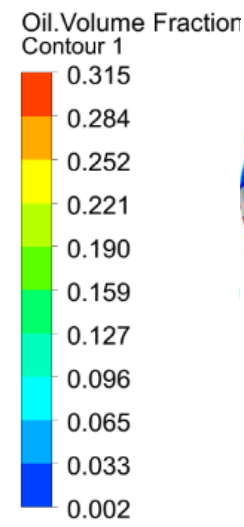

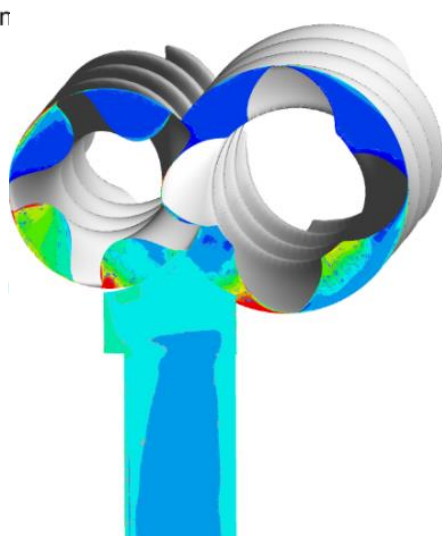

a

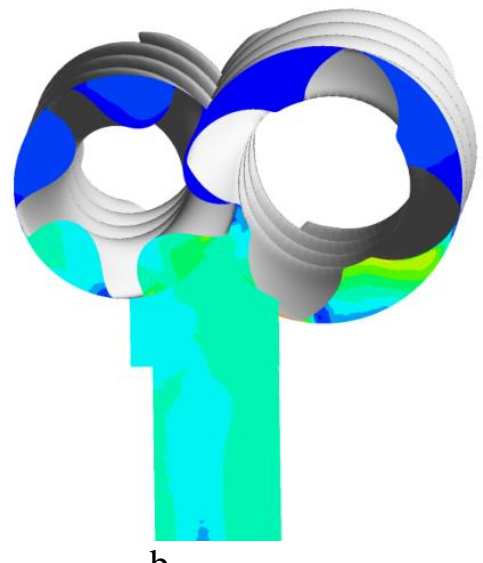

b

Fig. 5: Oil volume fraction contour near the discharge side for (a) inhomogeneous and (b) homogeneous distribution. 

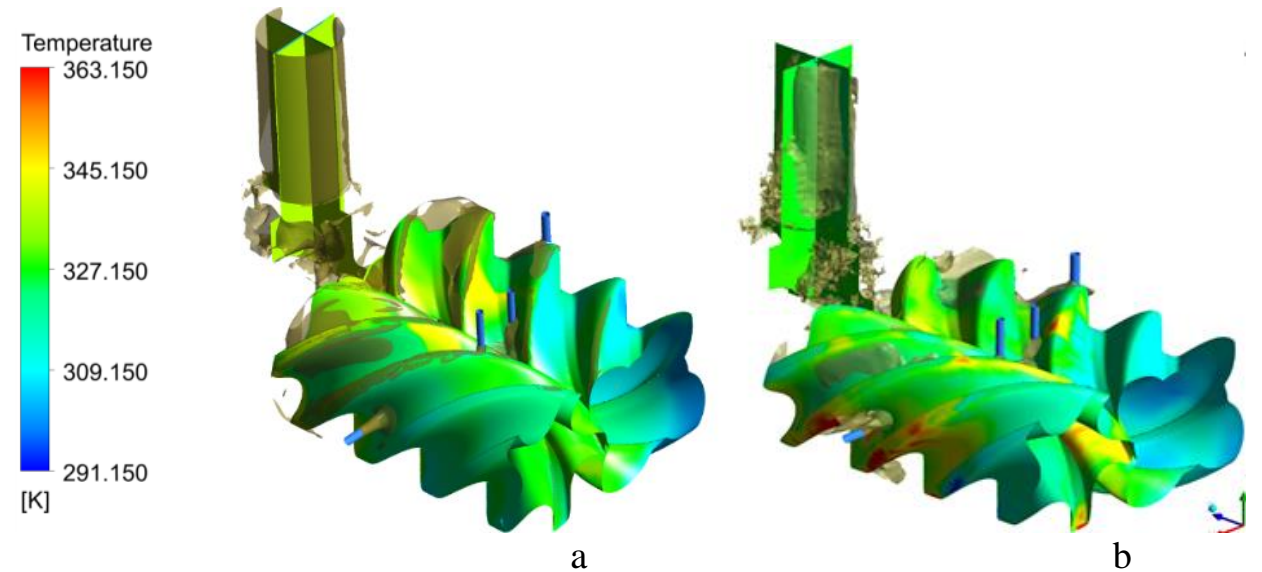

Fig. 6: Oil iso-surface with discharge temperature for (a) inhomogeneous and (b) homogeneous approach.

The velocity fields of oil and gas in the inhomogeneous model (Fig 6a) have different velocity fields and interphase drag and oil due to the inertial forces adheres on the rotor tips and casing walls. On the other hand, with homogeneous model shown in Fig. 6b, air and oil have the same velocity field in which case oil is not retained in the leakage gaps which leads to higher leakage flows. Finally, due to unrealistic oil distribution, local spots of significant temperature are observed near the rotor surface. This confirms that with homogeneous modelling, the main functions of oil which is to cool and seal the chamber is not very well captured.

\section{Conclusion}

In this research, a comparison is drawn between homogeneous and inhomogeneous multiphase model with ANSYS $\mathrm{CFX}$ solver using single rotor numerical grid generated by $\mathrm{SCORG}^{\mathrm{TM}}$. Use of homogeneous model reduces CPU time required by the solver which is around $63 \%$ of the computational time required for inhomogeneous model. However, the results predicted by the homogenous model are deviating from the experimental results more. The key conclusions are:

- Homogeneous model predicts lower mass flow rate by $30.6 \%$ and indicated power by $25.8 \%$ compared to measured data

- Inhomogeneous model predicts both flow rate and indicated power very close to the experimental data. Also, the distribution of oil, heat transfer between gas and oil and the sealing effects are well captured

- Though the homogeneous model requires shorter CPU time, it is much more unstable than the inhomogeneous which requires higher under relaxation factors in the solver and more efforts to achieve converged solution.

Overall, CFD modelling gives a clear visualisation on distribution of oil and temperature in the chamber. Therefore, this an effective tool to improve compressor design. In future, different multiphase modelling techniques such as VOF and segregated solvers can be explored to achieve better mass balance, reduced solving time and more accurate results.

\section{References}

[1] S. Rane, A. Kovacevic, and N. Stosic, "CFD Analysis of Oil Flooded Twin Screw Compressors," in 23rd International Compressor Engineering Conference, 2016.

[2] K. Hanjalic and N. Stosic, "Development and Optimization of Screw Machines With a Simulation Model-Part II: Thermodynamic Performance Simulation and Design Optimization," Journal of Fluids Engineering, vol. 119, no. 3, pp. 664-670, 1997.

[3] A. Kovacevic, "Three-Dimensional Numerical Analysis for Flow Prediction in Positive Displacement Screw Machines," City University London, 2002.

[4] A. Kovacevic, "Boundary adaptation in grid generation for CFD analysis of screw compressors," International Journal for Numerical Methods in Engineering, vol. 64, no. 3, pp. 401-426, 2005.

[5] A. Kovacevic, N. Stosic, and I. Smith, Screw Compressors: Three Dimensional Computational Fluid Dynamics and Solid Fluid Interaction. Springer Verlag, Berlin, 2005. 
[6] J. Voorde Vande and J. Vierendeels, "A Grid Manipulation Algorithm for ALE Calculations in Screw Compressors," in 17th AIAA Computational Fluid Dynamics Conference, 2005.

[7] I. Papes, J. Degroote, and J. Vierendeels, "3D CFD Analysis of an Oil Injected Twin Screw Expander," in Proceedings of the ASME 2013 International Mechanical Engineering Congress and Exposition IMECE2013.

[8] S. Rane and A. Kovacevic, "Advances in Engineering Software Algebraic generation of single domain computational grid for twin screw machines. Part I. Implementation," Advances in Engineering Software, vol. 107, pp. 38-50, 2017.

[9] A. Kovacevic and S. Rane, "Algebraic generation of single domain computational grid for twin screw machines Part II - Validation," Advances in Engineering Software, 2017.

[10] H. Ding and Y. Jiang, "CFD simulation of a screw compressor with oil injection," in 10th International Conference on Compressors and their Systems, no. 2017.

[11] C. W. Hirt and B. D. Nichols, "Volume of Fluid (VOF) Method for the Dynamics of Free Boundaries," vol. 225, pp. 201-225, 1981.

[12] B. Godderidge, S. Turnock, M. Tan, and C. Earl, "Computers \& Fluids An investigation of multiphase CFD modelling of a lateral sloshing tank," Computers and Fluids, vol. 38, no. 2, pp. 183-193, 2009.

[13] C. E. Brennen, "Fundamentals of Multiphase Flows," Technology, vol. 128, no. 1, pp. 1-18, 2005.

[14] “ANSYS CFX-Solver Theory Guide," 2006.

[15] S. Rane, A. Kovacevic, and N. Stosic, "Developments in Screw Machine Grid Generation and Application to Multiphase Flows," in 3rd Short Course and Forum - Computational Fluid Dynamics in Rotary Positive Displacement Machines, 2017. 